\title{
Diagnosis and treatment difficulties in the case of a patient with chronic Low Back Pain
}

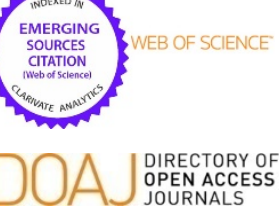

Adriana Lupu

${ }^{1}$ Medicine and Pharmacy Faculty, Galați

\begin{abstract}
Several definitions of the case study have been formulated over time, but in the following we will quote a definition that we consider appropriate for the clinical case that we will present, namely: "Case study is a method of direct confrontation of participants with a real, genuine situation, taken as a typical example, representative of a set of problematic situations and events that require a diagnosis and decision ". In reference to this definition, we emphasize that the importance of case presentations in medical practice is unquestionable as it brings to the attention of physicians other new or unusual, particular pathological conditions, difficulties or diagnostic errors, atypical clinical developments or unexpected treatment responses. The purpose of presenting this clinical case is to draw an alarm signal on the simplistic way in which the disease is still being addressed and not the patient in its entirety and to stimulate young doctors to review their own schemes and algorithms of clinical judgment. Particularities of the case to be presented are that the patient under investigation has about 10 months of intense hyper-lobular hyperplastic seizures with atypical development and, although presented to many specialists, the multiple co-morbidities of the patient have hampered the formulation of a diagnosis of certainty. Consequently, no adequate treatment has been established during this period to reduce its pain, so the patient's confidence in the medical act is now seriously altered.
\end{abstract}

Key words: Low Back Pain

\section{IMPORTANCE OF THE PROBLEM}

One person from eight suffers from degenerative disc disease, as well as from various joint diseases (arthrosis, arthritis, sciatica), the pain being located in the upper, medial or inferior part of the spine. At first, it is manifested as a slight redness, then pain occurs when walking or bending, and then installs a gradual rigidity that can affect the whole body and last but not least, the quality of life.

Statistical:

- WHO states that $85 \%$ of the world's population is at least once in the lifetime of experiencing a back pain

- These diseases can occur in both sexes, most often between the ages of 30 and 50; incidence increases with age

- It is the most common cause of functional impotence in patients $<45$ years

- It is the second most common neurological disease - after the headache and the most common cause of disability and job loss.

- It is the second cause of addressability in the family doctor's office

- $90 \%$ of the lumbar pain improves and disappears in 2 to 6 weeks, especially if the patients remain active

- The recurrence is frequent; $60-80 \%$ of patients have relapses over the next 2 years

\section{CASE PRESENTATION}

A 60-year-old, retired, senior, urban, married, physician has been presented to a physician for a sudden onset of hyperalgesic episodes in a highlumbar lumbar lobe that is interested in both the left hand chest, the left lobe, .

Algic episodes are intermittently accompanied by macroscopic hematuria.

Personal physiological antecedents - no matter what.

In the personal pathological history of the patient we remind:

- ischemic cardiopathy by bivascular damage;

- two acute myocardial infarctions treated by interventional arrhythmia for right and left obstruction with double stenosis;

- angina pectoris;

- repeated episodes of typical atrial flutter abraded;

- Stage II HTA, additional high risk;

- dyslipidemia;

- obesity;

- Type II diabetes mellitus treated with ADO;

- sensitive lime polyneuropathy;

- chronic stage II kidney disease;

- Multicular bilateral renal lithiasis with a $22 \mathrm{~mm}$ coraliform calculation. located in the stinger kidney basin. 
The heredo-collateral history highlights:

- Mother: obesity, HTA, coxarthrosis, dying rectosigmoidian neoplasm;

- Dad: Vertebro-basilar cerebral insufficiency with lacunarism episodes, HTA, who died as a result of cerebrovascular accident.

Risk Factors: Obesity (height $=170 \mathrm{~cm}$, weight $=$ $115 \mathrm{Kg})$, DZ type II, hypercholesterolemia, HTA stage II.

The history of the disease. The patient has about 10 months of dorsoclinic hyperalgic episodes that irradiate at the base of the posterior left anterior hemodialysis, with the interest of the stinger's flank, of the sting iliac fossa. and the hippodrome; intermittent, macroscopic hematuria episodes.

As the pains became more and more intense, the patient addressed the family doctor who, considering chronic kidney disease, led him to the urology service where he concluded that the pains were not of kidney and were prescribed antispasmodic and analgesic in times of haematuria, while reducing the dose of anticoagulants.

As the pain continues to appear in episodes that are more frequent and is aggravated by prolonged orthostatism, the patient has addressed the neurology service that has not provided a diagnosis of certainty and the recommended treatment (Gabaran) did not stop the algic passages, so the neurologist recommended rheumatologic consultation.

Following the CTs performed in October 2016 and April 2017, the patient was diagnosed with paramedian dissection protrusion at D3-D4 and dorsal cifoscoliosis with degenerative changes of the middle third disks.

The rheumatologist argues that classic antiinflammatory and anti-algic drugs are not appropriate due to heart disease and recommend kinetotherapeutic procedures.

After 15 sessions of combined procedures (electrical procedures, massage and acupuncture) applied with restraint due to heart disease, the patient had a short period of relief after which the pains returned with the same intensity. Later, electrical procedures were judged to be contraindicated due to suspicion of vertebral hemangioma.

From his personal history it is evident that for about 15 years the patient eliminates renal calculi of different sizes.
Under this condition, starting with 2014, the patient was diagnosed with renal macrocalculature of approximately $20 \mathrm{~mm}$. located in the stinger kidney basin, for which two lithotripsy sessions were performed without positive results.

It is also from personal history that the patient is known to be diabetic, hypertensive, with ischemic heart disease by bivascular disorder, myocardial infarction in the inferior (in 2008) revascularized by implantation of stent in the right coronary (in 2009). Following 4 episodes of autoimmune atrial fibrillation and typical antiretroviral atrial flutter converted to sinus rhythm, in 2015 ablation of the cavity-tricuspid isthm was achieved with the obstruction of the ismic bidirectional bloc.

In October 2016, due to anginal symptoms and positive effort test, it is decided to implant a new stent on the stng coronary.

Since then, the patient has received chronic treatment with anti-coagulants and anticoagulants and treatment for associated conditions.

The objective clinical examination highlights:

- normally conformed torso, no rallies, rhythmic heart rhythms, bradycardic, breathless, pulses present bilaterally to distances

- TA 140/80 mm.Hg., AV 46 bpm.

- volume-abdomen, with significant fat, painless palpation

- kidneys unblended; loja renala stg.- Giordano positive; normal bleeding

- thoraco-dorsal stg. on palpation is highlighted the important paravertebral muscular contracture;

- The mobility of the spine is limited, with evidence of an antalgic scoliosis

- the ODI score score for the 10 questionnaire categories reveals that patient pain is at the $35 \%$ ODI scale, which reveals a moderate disability

- on the analogue visual pain scale (VAS), the patient estimates that the pain is at an average daily level of about $55 \%$, and in hyperalgic episodes the pain reaches $85 \%$, which causes it to interrupt the activity

\section{Paraclinical and laboratory examinations:}

- glucose - 119 mg / dL; cholesterol - 178 mg / dL; HDL cholesterol - $47 \mathrm{mg}$ / dL; LDL cholesterol - 96 mg / dL; uric acid - $4.90 \mathrm{mg} / \mathrm{dL}$; total calcium $9.12 \mathrm{mg} . / \mathrm{dL}$; urea - $42 \mathrm{mg} / \mathrm{dL}$; creatinine - 1.20 $\mathrm{mg} . / \mathrm{dL}$; 
- the full urine test reveals 50 erythrocytes / IU; urinary sediment: 8-10 hematite, 3-4 leukocytes, amorphous urethra.

- Eco abdomen: liver with normal size, increased stentosis type ecogenity. Kid stg. shows the average caliceal a computational image of approximately 20 $\mathrm{mm}$, unobstructed. Colecist, pancreas, spleen, kidney as normal eco. Bladder with slightly thickened mucosa, no calculus. Prostate with upper limit dimensions.

- ECG: sinus rhythm; elements of SS.

- CT abdomino-pelvic revealed the presence of a basal coraliform calculus with a diameter of 22/15 $\mathrm{mm}$.

- The lumbar spine CT of October 2016 concluded: a moderate height decrease of the L5 - S1 disk with posterior disc disc.

- Cervical and thoracic CT of April 2017 reveals degenerative changes of all cervical disks, more pronounced at C5 - C6 and C6 - C7 levels. Protrusion disorder paramedian dr. To D3 - D4, dorsal cifoscolioza with degenerative changes of the middle third disks.

\section{Positive diagnosis}

Following investigations, the following diagnoses were formulated:

1. Dorsolombalgie stg. chronic, with painful activation and hyperalgic episodes, multifactorial vertebral - discogenic, stage III, stage I, irritation and viscerogenic (coraliform stalk calculus).

2. Morbid obesity.

3. Chronic ischemic coronary heart disease.

4. Type II diabetes mellitus (in treatment with oral antidiabetic agents).

5. Hyperlipoproteinemia (in statin therapy).

6. Bilateral kidney ligation.

Chronic mixed anticoagulation.

8. In observation - vertebral hemangioma at L1.

\section{Differential diagnosis}

Although the positive diagnosis is well argued and supported by both the objective examination and the paraclinical and laboratory explorations due to the algal bivalent vertebro-discogenic and viscerogenic component, the differential diagnosis is made with the following disorders:

- Ankylosing spondylitis

- Spondilartroza

- Hernia de disc

- Osteoporosis, osteomalacia

- Paget deformity osteitis
- metastasis

- plasmacytoma

- Infectious diseases (osteomyelitis,

spondylodisitis)

- Irradiated pains in the abdominal area (aortic aneurysm, gastrointestinal disorders)

- Renal disorders (infections, calculus, traumatic bleeding)

- Trauma to the column

The disease progression is stationary, with recurrent hyperalgesic episodes, poorly influenced by prescribed anti-algic and antispasmodic medication. Immediate prognosis is reserved due to associated conditions, and the long-term prognosis is dependent on:

- elimination of the visceral algic cause (removal of renal calculus from stinger kidney)

- the vertebro-discogenetic algal component will be treated according to an algorithm established by an interdisciplinary team (cardiologist rheumatologist - neurologist - physical therapist).

\section{Recommendations:}

1. Slow weight loss

2. Respect hygiene-postural instructions

3. Balneo-climatic treatment twice a year

Physical and kinetological procedures for drossolumbar algo-dysfunction: dry massage, hydrokinetotherapy, kinetotherapy (Williams program the first set of exercises)

5. During hyperalgic periods, the patient is advised to rest in bed in a relaxing position that relieves pain, local massage with anti-inflammatory ointments (Dicloreum, Deep Relief), antialgic medication allowed by associated conditions (Piafen, Tramal)

6 . For the affection of the spine, the patient will receive the following medication: MSM - $750 \mathrm{mg}$. $2 \mathrm{cp} /$ day for one month, Ala 600 SOD 1cp / day for 14 days

7. The cardiologist will appreciate the possibility of discontinuing anticoagulant and antiaggregant medication for surgery to eliminate stg baseline calculus. 


\section{Discusion and conclusions}

Although the patient addressed several physicians in different medical fields, they did not have an overview of the patient's overall pathology, the case management being flawed.

Thus, each specialist has approached unilaterally pain, through his specialty, without regard to associated affections. This therapeutic attitude, sometimes at risk for the patient, resulted in the perpetuation of the symptoms and the tremendous evolution of the disease, and finally, the pain was not improved for almost a year, contributed to altering the patient's confidence in the medical act . Unfortunately, "standardized medicine" with standard diagnostics is still practiced, to the detriment of the modern concept of patient diagnosis and treatment within an interdisciplinary team.

Given the unpredictable nature of medicine and the fact that some patients experience unusual clinical conditions, develop atypical, or respond to therapeutic interventions that have not been previously described, young doctors will have to admit that only teamwork can provide solutions to the patient's problems, so that the trust in the medical act is complete.

\section{Bibliography:}

1. http://healingchronicpain.org/content/backpain/causes.asp

2. http://www.painphisicianjournal.com/linkout_vw.php

3. http://www.patient.co.uk/helth/nonspecific-lower-backpain-in-adults

4. Nicolescu 0. (coord.) - "Management aplicativ - studii de caz", Bucuresti, Institutul Manager, 1994, pag. 7 - 10

5. Voiculescu M - "Redactarea unui caz clinic", Viata medicala, 1979, XVII,183

6. Voiculescu M - "Importanta cazuisticii clinice", Viata medicala, 1984, XXXI, 481 